\title{
Fetal Diagnosis of Left Ventricular Aneurysm: A Case Report
}

\author{
Lauren Giacobbe, M.D., ${ }^{1}$ Preston Williams, M.D., ${ }^{1}$ Kirk Ramin, M.D., ${ }^{1}$ \\ and Shanthi Sivanandam, M.D. ${ }^{2}$
}

Congenital left ventricular aneurysm is a rare but potentially lethal condition. We describe a case of isolated congenital left ventricular aneurysm diagnosed at 28 weeks' gestation. In addition to standard imaging, we utilized color-coded transthoracic tissue Doppler techniques to further evaluate the aneurysm postnatally.

KEYWORDS: Fetal echocardiography, ventricular aneurysm, congenital heart disease, ultrasound

Congenital left ventricular aneurysm is a rare but potentially lethal cardiac anomaly. We describe a case of isolated congenital left ventricular aneurysm diagnosed at 28 weeks' gestation. Standard diagnostic techniques were augmented with color-coded transthoracic tissue Doppler imaging to further evaluate the aneurysm postnatally.

\section{CASE REPORT}

A 22-year-old woman, gravida 4, para 2, was referred for an abnormal cardiac finding on a comprehensive obstetric ultrasound performed at 28 weeks' gestation. Due to late prenatal care, this was the patient's first ultrasound in the pregnancy. The left ventricle of the fetus was described as having an hourglass-like shape, and there was concern for irregular contractility. No other fetal abnormalities were identified, and there was no evidence of fetal hydrops. The patient had no significant past medical history and denied exposure to teratogens during the pregnancy. She did report symptoms consistent with a viral illness at 5 to 6 weeks' gestation.

A fetal echocardiogram demonstrated a discrete segment of the apical left ventricular wall that exhibited a thin-walled outpouching with a wide communication to the left ventricular cavity (Fig. 1). The left ventricular volume and the aneurysm were measured by tracing the endocardium using a single-plane ellipse calculation. The left ventricular aneurysm measured $11 \times 13 \mathrm{~mm}$ in diameter, and the neck of the aneurysm measured $5 \mathrm{~mm}$. The aneurysm volume in diastole was $1.1 \mathrm{~mL}$. The ratio of aneurysm volume to left ventricular volume in diastole was 0.5 , and the left ventricular myocardial performance index was 0.4. The measurements from subsequent echocardiograms are reported in Table 1. Color flow imaging showed abnormal low-velocity turbulent flow in the pouch, but there was no demonstrable contraction during systole. None of the prenatal echocardiograms revealed thrombus within the aneurysm, arrhythmia, or pericardial effusion. The mitral valve apparatus consistently appeared normal, and there was no mitral insufficiency noted.

An elective induction and delivery occurred at 39 weeks' gestation. The infant weighed 6 pounds, 11 ounces. Apgar scores were 9 and 9 at 1 and 5 minutes, respectively. The infant was transferred to the neonatal intensive care unit for further evaluation. A postnatal

Departments of ${ }^{1}$ Obstetrics, Gynecology, and Women's Health, and

${ }^{2}$ Pediatrics, University of Minnesota, Minneapolis, Minnesota.

Address for correspondence and reprint requests: Shanthi Sivanandam, M.D., Department of Pediatrics, University of Minnesota, 420 Delaware Street.SE MMC 94, Minneapolis, MN 55455 (e-mail: silv0099@umn.edu).

Am J Perinatol Rep 2011;1:91-94. Copyright (C) 2011 by Thieme

Medical Publishers, Inc., 333 Seventh Avenue, New York, NY 10001, USA. Tel: +1 (212) 584-4662.

Received: March 24, 2011. Accepted after revision: May 12, 2011. Published online: July 22, 2011.

DOI: http://dx.doi.org/10.1055/s-0031-1284221.

ISSN 2157-6998. 


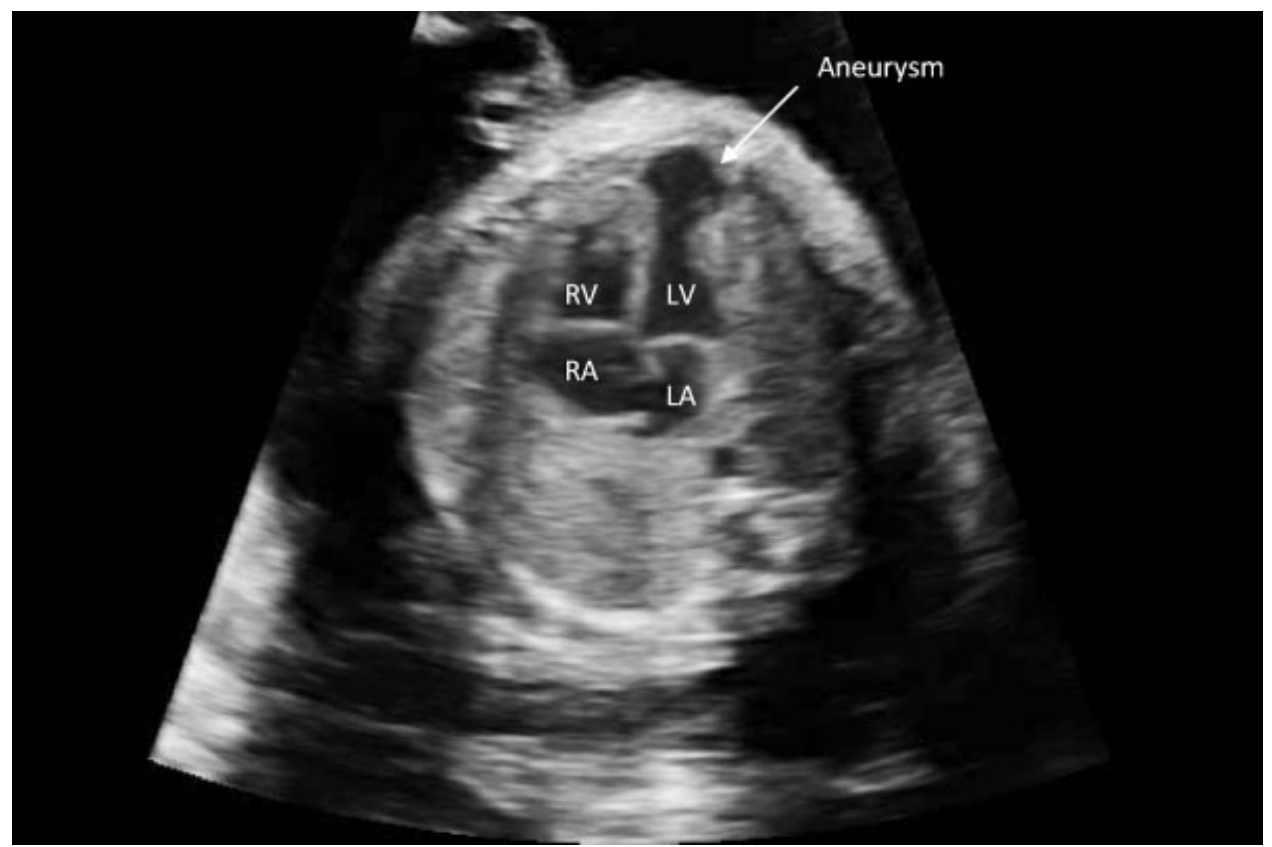

Figure 1 Abnormal four-chamber view on ultrasound demonstrating the left ventricular aneurysm. LA, left atrium; LV, left ventricle; $R A$, right atrium; $R V$, right ventricle.

transthoracic echocardiogram confirmed the diagnosis of left ventricular aneurysm and demonstrated otherwise normal intracardiac anatomy. To demonstrate the akinetic segment of the aneurysm, we utilized tissue Doppler imaging (TDI), illustrating a color-coded measure of myocardial velocity. Figure 2 demonstrates the normal myocardial velocity across the left ventricular wall (represented by the yellow and aqua colors), as well as the akinetic segment in the aneurysm (represented by the red, green, and orange colors). The TDI myocardial velocity in the normal myocardium was significantly different compared with the velocity in the akinetic aneurysm.

The infant is currently 3 months old and remains asymptomatic. There is no evidence of congestive heart failure, arrhythmia, or thrombus. The infant was placed on chronic aspirin as a therapeutic measure to prevent thromboembolism.

\section{DISCUSSION}

The advent of ultrasound and echocardiography has led to earlier detection of congenital ventricular diverticu- lum and aneurysm. Both entities appear to be due to developmental anomalies involving the left ventricular myocardium, thought to result from a focal weakening of the ventricular wall. Fetal viral infection and myocardial ischemia have been considered possible etiologies ${ }^{1}$; however, a review of seven cases by Papagiannis et al did not support these theories. ${ }^{2}$ Because most cases are asymptomatic, the incidence is unknown; however, congenital ventricular aneurysm is estimated to occur in 0.5 per 100,000 live births. ${ }^{3}$ Although the distinction between ventricular aneurysm and ventricular diverticulum remains inconsistent, it is important to distinguish the two entities, because the treatment and prognosis can differ. The classification criteria include connection to the ventricular cavity, wall composition, wall motion, and association with intracardiac abnormalities or midline defects. $^{3-6}$

An aneurysm implies a wide communication with the ventricle. The aneurysm can be composed of fibrous, thinned myocardium or it can present as an outpouching through disrupted myocardium. ${ }^{3,6}$ Due to these histological findings, the aneurysm is noncontractile during

Table 1 Congenital LV Aneurysm Measurements

\begin{tabular}{llll}
\hline & 28-wk GA & 32-wk GA & Birth \\
\hline Diameter of aneurysm (mm) & $11 \times 13$ & $11 \times 13$ & $12 \times 13$ \\
Neck of aneurysm (mm) & 5 & 7 & 7 \\
Volume of aneurysm (mL) & 1.1 & 1.4 & 7 \\
AV/LV volume & 0.5 & 0.4 & 1.8 \\
Myocardial performance index & 0.4 & 0.5 & 0.2 \\
\hline
\end{tabular}

$\mathrm{AV} / \mathrm{LV}$ volume is the ratio of aneurysm volume to left ventricular volume in diastole. GA, gestational age. 


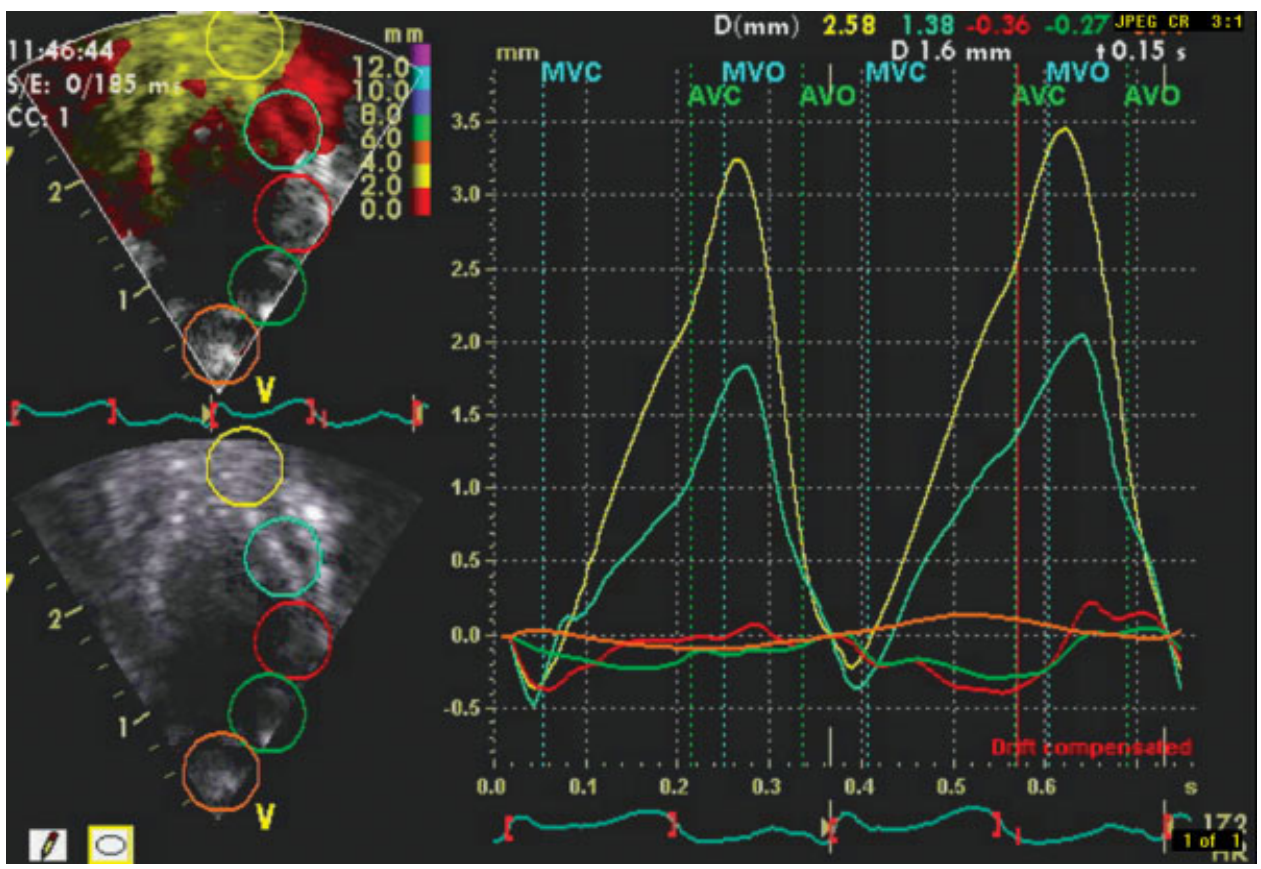

Figure 2 Transthoracic color-coded tissue Doppler image of the normal myocardial velocity across the left ventricular wall (represented by the yellow and aqua colors), as well as the akinetic segment in the aneurysm (represented by the red, green, and orange colors).

systole. Although the aneurysm may arise from the ventricular septum, the wall, or the apical region, apical aneurysm are the most common. ${ }^{2}$ Congenital left ventricular aneurysms are not typically associated with other intracardiac anomalies. ${ }^{7}$ Complications include spontaneous rupture, arrhythmia, congestive heart failure, thromboembolism, and a mass effect resulting in underdevelopment of the lungs. ${ }^{2,8}$ Fatal cardiovascular complications can occur in the neonatal period. ${ }^{9}$

A diverticulum involves a narrow connection with the ventricular cavity and is of similar thickness and layering as the rest of the myocardium. Systolic contractility is a constant characteristic of congenital ventricular diverticulum. ${ }^{9}$ The diverticulum usually extends into the pericardial space. Although nonapical congenital ventricular diverticula tend to be isolated findings, apical lesions have been associated with a syndrome of midline thoracoabdominal defects and with other intracardiac malformations. ${ }^{9}$

The optimal treatment of congenital ventricular diverticula and congenital ventricular aneurysms remains unclear. When symptomatic, or when associated with other cardiac abnormalities, surgical treatment is usually recommended. ${ }^{9}$ According to a review article by Marijon et al, the prognosis for congenital ventricular diverticulum depends on associated intracardiac malformations, but is generally favorable after repair. ${ }^{9}$ The prognosis for congenital ventricular aneurysm is related to size, location, degree of mitral valve involvement, and ventricular arrhythmias.
During routine second-trimester ultrasound examinations, the four-chamber view of the heart is essential to aid in the diagnosis of congenital heart disease. If an abnormality is detected, echocardiography is the standard modality to further evaluate the fetal heart. In our case, we utilized transthoracic color-coded tissue Doppler imaging to demonstrate the akinetic segment of the aneurysm. TDI offers a more detailed postnatal examination of the heart and the ability to evaluate myocardial velocity. We believe it is a useful modality that can assist in the diagnosis of and distinction between congenital ventricular aneurysms and diverticula.

\section{REFERENCES}

1. Gembruch U, Steil E, Redel DA, Hansmann M. Prenatal diagnosis of a left ventricular aneurysm. Prenat Diagn 1990;10: 203-209

2. Papagiannis J, Van Praagh R, Schwint O, et al. Congenital left ventricular aneurysm: clinical, imaging, pathologic, and surgical findings in seven new cases. Am Heart J 2001;141: 491-499

3. Del Río M, Martínez JM, Bennasar M, et al. Prenatal diagnosis of a right ventricular diverticulum complicated by pericardial effusion in the first trimester. Ultrasound Obstet Gynecol 2005; 25:409-411

4. Prefumo F, Bhide A, Thilaganathan B, Carvalho JS. Fetal congenital cardiac diverticulum with pericardial effusion: two cases with different presentations in the first trimester of pregnancy. Ultrasound Obstet Gynecol 2005;25:405-408 
5. Arenas Ramírez J, Fernandez Castro C, Otero Chouza M, Duplá Parugues B, Montes Sanchez R. Persistent and redundant eustachian valve simulating atrial tumor: prenatal diagnosis. Ultrasound Obstet Gynecol 2007;29:704-707

6. McAuliffe FM, Hornberger LK, Johnson J, Chitayat D, Ryan G. Cardiac diverticulum with pericardial effusion: report of two new cases treated by in-utero pericardiocentesis and a review of the literature. Ultrasound Obstet Gynecol 2005; 25:401-404
7. Chaubal N, Dighe M, Shah M, Chaubal J, Raghavan J. Congenital left ventricular aneurysm: prenatal sonographic diagnosis. J Ultrasound Med 2004;23:125-128

8. Pipitone S, Sperandeo V, Mongiovi M, Roberto G, Centineo G. Prenatal diagnosis of ventricular aneurysm: a report of two cases and a review. Prenat Diagn 2002;22:131-136

9. Marijon E, Ou P, Fermont L, et al. Diagnosis and outcome in congenital ventricular diverticulum and aneurysm. J Thorac Cardiovasc Surg 2006;131:433-437 